\title{
Statistical analysis of control quality of MPC using testing hypothesis
}

\author{
Marek Kubalcik ${ }^{1, *}$, Vladimir Bobal ${ }^{1}$, and Tomas Barot $^{2}$ \\ ${ }^{1}$ Department of Process Control, Tomas Bata University in Zlin, Faculty of Applied Informatics, Nad Stranemi 4511, 76005 Zlin, Czech \\ Republic \\ ${ }^{2}$ Department of Mathematics with Didactics, University of Ostrava, Faculty of Education, Fr. Sramka 3, 70900 Ostrava, Czech Republic
}

\begin{abstract}
Methods of the statistical induction have a significant role in the quantitative research. In a wide spectrum of research areas, the methods based on testing hypotheses have been frequently used. However, in the area of the process control, testing hypothesis has not been widely considered as an established tool for signal analyses, although signals in control loops are suitable for analysis by means of quantitative statistical methods due to their stochastic character. Particularly, a statistical paired comparison can be applied for analysis of control quality achieved with different control algorithms. This comparison can be based on a paired comparison of corresponding signals obtained with different or modified control algorithms. The aim of this paper is a proposal of incorporation of testing hypothesis to analysis of control quality. The analysis was performed on a strictly defined significance level 0.001 , which is a standardly used value in technical applications. As an example was demonstrated analysis of control quality achieved with two versions of a predictive controller. Finally, achieved results of paired comparison using testing hypothesis are discussed.
\end{abstract}

\section{Introduction}

One of the main aims in the process control [1] is achievement of a suitable quality of control. The quality of control is often examined in order to evaluate which control algorithm reaches the best results in a particular control problem. The control algorithms which yield appropriate control results are often complex and computationally demanding. Therefore, there is an effort to propose modifications of the control algorithms in order to simplify them. These simplifications are obviously at the expense of the control quality. The quality of the control is then examined in order to evaluate whether the modified control algorithms are still suitable for a particular control problem or not. In this paper, this case was considered.

As a suitable example of an improving a control algorithm with regards to decreasing of the computational complexity, a multivariable Model predictive control (MPC) will be considered. Model predictive control is one of currently utilized modern control methods, as can be seen in e.g. [2]-[4].

With regards to high computational requirements caused by multivariability of the controlled process, higher prediction and control horizons and number of constraints of variables, modifications of MPC control algorithm are advantageous as has been proposed e.g. in [5]. A significantly important part of the constrained MPC is an optimization task. It is characterized by higher computational complexity. Therefore, a reduction of the computational complexity of the optimization methods in MPC has been widely researched [6]. An application of an appropriate numerical method is necessary for achievement of the vector of future increments of the manipulated variables given by solving the optimization problem incorporating a suitable cost function with considered constraints. As frequently used numerical method for solving the optimization problem in MPC, the Hildreth method has been widely used [7]. Besides modifications of the numerical optimization methods, general simplified optimization strategies used in MPC controllers have been proposed [6].

The control quality is mostly analyzed using general control quality criterions based on sums of powers of control increments and on sums of powers of control errors [8]. These criterions can result only in descriptive attributes of control quality. Therefore, on the basis of particular values of the criterions, it is not possible to identify if the control quality achieved with one algorithm is statistically significantly different from control quality achieved with another algorithm.

The aim of this paper is to examine the control quality with use of testing hypotheses [9]-[10] on existence of statistically significant differences between partial values of signals measured in individual sampling periods. The analysed signals were controlled outputs, increments of manipulated variables, control errors and manipulated variables. The values of the signals can be paired compared by the Paired T-test or by the Wilcoxon Paired test. If the values of the signals have normal

\footnotetext{
* Corresponding author: $\underline{\text { kubalcik@utb.cz }}$
} 
probability distribution character, then the Paired T-test has to be applied. In the opposite case, the Wilcoxon Paired test should be used. An important assumption for testing hypotheses is a declaration of a significance level $\alpha$, which is considered as strictly defined for 0.001 value in the technical applications. The analysis was then performed on this strictly defined significance level.

In this paper, the testing hypothesis is applied in comparison of control quality between a standard MPC algorithm and particularly modified MPC algorithm [11], where a modification of an iterative numerical optimization method was proposed. The modification consists in an addition of a new termination condition in the iterative algorithm. In this paper, the obtained results of the analysis using testing hypothesis are more mathematically supported then the simple descriptive values of the control quality criterions.

\section{Multivariable MPC analyzed by standard control quality criterions}

In MPC, the control quality criterions $J_{1}(1)$ and $J_{2}(2)$ will be evaluated as well as a number of floating point operations. The floating point operations (flops) are measured both for the MPC algorithm without the modifications $(F)$ and for the modified MPC algorithm $\left(F^{*}\right)$, which was proposed in [11] (Section 3). The numbers of flops is determined using rules published in [12]. This standard analysis will be compared with our new proposal of the analysis of signals using testing hypothesis with regards to control quality in Section 4.

$$
\begin{gathered}
J_{1}=\sum_{k}\left[\Delta u_{1}(k)\right]^{2}+\sum_{k}\left[\Delta u_{2}(k)\right]^{2} \\
J_{2}=\sum_{k}\left[w_{1}(k)-y_{1}(k)\right]^{2}+\sum_{k}\left[w_{2}(k)-y_{2}(k)\right]^{2}
\end{gathered}
$$

A model with two inputs and two outputs (TITO) was further considered in the framework of MPC [1]-[4] in this paper.

The TITO system can be further expressed as (3)-(5) in the form of a general transfer matrix (3).

$$
\begin{gathered}
\boldsymbol{G}(z)=\left[\begin{array}{ll}
G_{11}(z) & G_{12}(z) \\
G_{21}(z) & G_{22}(z)
\end{array}\right] \\
\boldsymbol{Y}(z)=\boldsymbol{G}(z) \boldsymbol{U}(z) \\
\boldsymbol{U}(z)=\left[u_{1}(z), u_{2}(z)\right]^{T}, \boldsymbol{Y}(z)=\left[y_{1}(z), y_{2}(z)\right]^{T}
\end{gathered}
$$

The transfer matrix (3) can be transcribed to the form (6) of the matrix fraction.

$$
\boldsymbol{G}(z)=\boldsymbol{A}^{-1}\left(z^{-1}\right) \boldsymbol{B}\left(z^{-1}\right)=\boldsymbol{B}_{1}\left(z^{-1}\right) \boldsymbol{A}_{1}^{-1}\left(z^{-1}\right)
$$

The model can be also written in form (7)-(9) Polynomials of the second degree were considered.

$$
\boldsymbol{A}\left(z^{-1}\right) \boldsymbol{Y}(z)=\boldsymbol{B}\left(z^{-1}\right) \boldsymbol{U}(z)
$$

$$
\begin{gathered}
\boldsymbol{A}\left(z^{-1}\right)=\left[\begin{array}{cc}
1+a_{1} z^{-1}+a_{2} z^{-2} & a_{3} z^{-1}+a_{4} z^{-2} \\
a_{5} z^{-1}+a_{6} z^{-2} & 1+a_{7} z^{-1}+a_{8} z^{-2}
\end{array}\right] \\
\boldsymbol{B}\left(z^{-1}\right)=\left[\begin{array}{cc}
b_{1} z^{-1}+b_{2} z^{-2} & b_{3} z^{-1}+b_{4} z^{-2} \\
b_{5} z^{-1}+b_{6} z^{-2} & b_{7} z^{-1}+b_{8} z^{-2}
\end{array}\right]
\end{gathered}
$$

A widely used model in model predictive control is the CARIMA model which we can obtain by adding a disturbance model (10)-(11), where polynomial matrix $\boldsymbol{C}$ will be further supposed to be equal to the identity matrix.

$$
\begin{gathered}
\boldsymbol{A}\left(z^{-1}\right) \boldsymbol{y}(k)=\boldsymbol{B}\left(z^{-1}\right) \boldsymbol{u}(k)+\boldsymbol{C}\left(z^{-1}\right) \boldsymbol{\Delta}^{-1}\left(z^{-1}\right) \boldsymbol{n}(k) \\
\boldsymbol{\Delta}\left(z^{-1}\right)=\left[\begin{array}{cc}
1-z^{-1} & 0 \\
0 & 1-z^{-1}
\end{array}\right]
\end{gathered}
$$

The difference equations (10) of the CARIMA model are used for computation of predictions in predictive control. These equations can be further written into a matrix form (12)-(15).

$$
\begin{aligned}
& y_{1}(k+1)=\left(1-a_{1}\right) y_{1}(k)+\left(a_{1}-a_{2}\right) y_{1}(k-1)+a_{2} y_{1}(k-2)- \\
& -a_{3} y_{2}(k)+\left(a_{3}-a_{4}\right) y_{2}(k-1)+a_{4} y_{2}(k-2)+ \\
& +b_{1} \Delta u_{1}(k)+b_{2} \Delta u_{1}(k-1)+b_{3} \Delta u_{2}(k)+b_{4} \Delta u_{2}(k-1) \\
& y_{2}(k+1)=\left(1-a_{7}\right) y_{2}(k)+\left(a_{7}-a_{8}\right) y_{2}(k-1)+a_{8} y_{2}(k-2)- \\
& -a_{5} y_{1}(k)+\left(a_{5}-a_{6}\right) y_{1}(k-1)+a_{6} y_{1}(k-2)+ \\
& +b_{5} \Delta u_{1}(k)+b_{6} \Delta u_{1}(k-1)+b_{7} \Delta u_{2}(k)+b_{8} \Delta u_{2}(k-1) \\
& \boldsymbol{y}(k+1)=\boldsymbol{A}_{1} \boldsymbol{y}(k)+\boldsymbol{A}_{2} \boldsymbol{y}(k-1)+\boldsymbol{A}_{3} \boldsymbol{y}(k-2)+ \\
& +\boldsymbol{B}_{1} \Delta \boldsymbol{u}(k)+\boldsymbol{B}_{2} \Delta \boldsymbol{u}(k-1) \\
& \boldsymbol{A}_{1}=\left[\begin{array}{ll}
1-a_{1} & -a_{3} \\
-a_{5} & 1-a_{7}
\end{array}\right], \boldsymbol{A}_{2}=\left[\begin{array}{ll}
a_{1}-a_{2} & a_{3}-a_{4} \\
a_{5}-a_{6} & a_{7}-a_{8}
\end{array}\right] \\
& \boldsymbol{A}_{3}=\left[\begin{array}{ll}
a_{2} & a_{4} \\
a_{6} & a_{8}
\end{array}\right], \boldsymbol{B}_{1}=\left[\begin{array}{ll}
b_{1} & b_{3} \\
b_{5} & b_{7}
\end{array}\right], \boldsymbol{B}_{2}=\left[\begin{array}{ll}
b_{2} & b_{4} \\
b_{6} & b_{8}
\end{array}\right]
\end{aligned}
$$

It is necessary to directly compute three steps-ahead predictions by establishing of previous predictions to later predictions. Three past values of the system output are determined by computation:

$$
\begin{aligned}
& \hat{\boldsymbol{y}}(k+1)=\boldsymbol{A}_{1} \boldsymbol{y}(k)+\boldsymbol{A}_{2} \boldsymbol{y}(k-1)+\boldsymbol{A}_{3} \boldsymbol{y}(k-2)+ \\
& +\boldsymbol{B}_{1} \Delta \boldsymbol{u}(k)+\boldsymbol{B}_{2} \Delta \boldsymbol{u}(k-1) \\
& \hat{\boldsymbol{y}}(k+2)=\boldsymbol{A}_{1} \boldsymbol{y}(k+1)+\boldsymbol{A}_{2} \boldsymbol{y}(k)+\boldsymbol{A}_{3} \boldsymbol{y}(k-1)+ \\
& +\boldsymbol{B}_{1} \Delta \boldsymbol{u}(k+1)+\boldsymbol{B}_{2} \Delta \boldsymbol{u}(k) \\
& \hat{\boldsymbol{y}}(k+3)=\boldsymbol{A}_{1} \boldsymbol{y}(k+2)+\boldsymbol{A}_{2} \boldsymbol{y}(k+1)+\boldsymbol{A}_{3} \boldsymbol{y}(k)+ \\
& +\boldsymbol{B}_{1} \Delta \boldsymbol{u}(k+2)+\boldsymbol{B}_{2} \Delta \boldsymbol{u}(k+1)
\end{aligned}
$$

Computation of the predictions can be divided into recursion of the free response and recursion of the matrix 
of dynamics. The free response vector can be expressed as (19)-(25).

$$
\begin{aligned}
& \boldsymbol{y}_{0}=\left[\begin{array}{ll}
p_{11} & p_{12} \\
p_{21} & q_{22} \\
\hline p_{31} & p_{32} \\
p_{41} & p_{42} \\
\hline p_{51} & p_{52} \\
p_{61} & p_{62}
\end{array}\right]\left[\begin{array}{ll|ll|ll}
q_{11} & q_{12} & q_{13} & q_{14} & q_{15} & q_{16} \\
q_{21} & q_{22} & q_{23} & q_{24} & q_{25} & q_{26} \\
\hline q_{31} & q_{32} & q_{33} & q_{34} & q_{35} & q_{36} \\
q_{41} & q_{42} & q_{43} & q_{44} & q_{45} & q_{46} \\
\hline q_{51} & q_{52} & q_{53} & q_{54} & q_{55} & q_{56} \\
q_{61} & q_{62} & q_{63} & q_{64} & q_{65} & q_{66}
\end{array}\right]\left[\begin{array}{c}
y_{1}(k) \\
y_{2}(k) \\
y_{2}(k-1) \\
y_{2}(k-1) \\
y_{1}(k-2) \\
y_{2}(k-2)
\end{array}\right]= \\
& =\left[\begin{array}{l}
\boldsymbol{P}_{1} \\
\boldsymbol{P}_{2} \\
\boldsymbol{P}_{3}
\end{array}\right] \Delta \boldsymbol{u}(k-1)+\left[\begin{array}{lll}
\boldsymbol{Q}_{11} & \boldsymbol{Q}_{12} & \boldsymbol{Q}_{13} \\
\boldsymbol{Q}_{21} & \boldsymbol{Q}_{22} & \boldsymbol{Q}_{23} \\
\boldsymbol{Q}_{31} & \boldsymbol{Q}_{32} & \boldsymbol{Q}_{33}
\end{array}\right]\left[\begin{array}{c}
\boldsymbol{y}(k) \\
\boldsymbol{y}(k-1) \\
\boldsymbol{y}(k-2)
\end{array}\right]=\boldsymbol{P} \Delta \boldsymbol{u}(k-1)+\boldsymbol{Q}\left[\begin{array}{c}
\boldsymbol{y}(k) \\
\boldsymbol{y}(k-1) \\
\boldsymbol{y}(k-2)
\end{array}\right]
\end{aligned}
$$

$$
\boldsymbol{P}_{4}=\left[\begin{array}{ll}
p_{71} & p_{72} \\
p_{81} & p_{82}
\end{array}\right]=\boldsymbol{A}_{1} \boldsymbol{P}_{31}+\boldsymbol{A}_{2} \boldsymbol{P}_{21}+\boldsymbol{A}_{3} \boldsymbol{P}_{11}
$$

$$
\boldsymbol{Q}_{41}=\left[\begin{array}{ll}
q_{71} & q_{72} \\
q_{81} & q_{82}
\end{array}\right]=\boldsymbol{A}_{1} \boldsymbol{Q}_{31}+\boldsymbol{A}_{2} \boldsymbol{Q}_{21}+\boldsymbol{A}_{3} \boldsymbol{Q}_{11}
$$$$
\boldsymbol{Q}_{42}=\left[\begin{array}{ll}
q_{73} & q_{74} \\
q_{83} & q_{84}
\end{array}\right]=\boldsymbol{A}_{1} \boldsymbol{Q}_{32}+\boldsymbol{A}_{2} \boldsymbol{Q}_{22}+\boldsymbol{A}_{3} \boldsymbol{Q}_{12}
$$$$
\boldsymbol{Q}_{43}=\left[\begin{array}{ll}
q_{75} & q_{76} \\
q_{85} & q_{86}
\end{array}\right]=\boldsymbol{A}_{1} \boldsymbol{Q}_{33}+\boldsymbol{A}_{2} \boldsymbol{Q}_{23}+\boldsymbol{A}_{3} \boldsymbol{Q}_{13}
$$

$$
\boldsymbol{G} \Delta \boldsymbol{u}=\left[\begin{array}{cc|cc}
g(1,1) & g(1,2) & 0 & 0 \\
g(2,1) & g(2,2) & 0 & 0 \\
\hline g(3,1) & g(3,2) & g(1,1) & g(1,2) \\
g(4,1) & g(4,2) & g(2,1) & g(2,2) \\
\hline g(5,1) & g(5,2) & g(3,1) & g(3,2) \\
g(6,1) & g(6,2) & g(4,1) & g(4,2)
\end{array}\right]\left[\begin{array}{c}
\Delta u_{1}(k) \\
\Delta u_{2}(k) \\
\hline \Delta u_{1}(k+1) \\
\Delta u_{2}(k+1)
\end{array}\right]=
$$

$$
\begin{gathered}
=\left[\begin{array}{cc}
G(1,1) & 0 \\
G(2,1) & G(1,1) \\
G(3,1) & G(2,1)
\end{array}\right]\left[\begin{array}{c}
\Delta \boldsymbol{u}(k) \\
\Delta \boldsymbol{u}(k+1)
\end{array}\right] \\
\boldsymbol{G}_{41}=\left[\begin{array}{ll}
g_{71} & g_{72} \\
g_{81} & g_{82}
\end{array}\right]=\boldsymbol{A}_{1} \boldsymbol{G}_{31}+\boldsymbol{A}_{2} \boldsymbol{G}_{21}+\boldsymbol{A}_{3} \boldsymbol{G}_{11}
\end{gathered}
$$

If the control horizon is lower than the prediction horizon a number of columns in the matrix is reduced. Predictions can be written in a compact matrix form (26).

$$
\begin{aligned}
& \hat{\boldsymbol{y}}(k+j)=\boldsymbol{G} \Delta \boldsymbol{u}(k+j-1)+\boldsymbol{P} \Delta \boldsymbol{u}(k-1)+\boldsymbol{Q} \boldsymbol{y}(k-j+1) \\
& j \leq N
\end{aligned}
$$

The computation of a control law of MPC is based on minimization of quadratic cost function (27) related to quadratic programming [13].

$$
J(k)=\sum_{j=1}^{N} \boldsymbol{e}(k+j)^{2}+\lambda \sum_{j=1}^{N_{u}} \Delta \boldsymbol{u}(k+j)^{2}
$$

$\boldsymbol{e}(k+j)$ is a vector of predicted control errors, $\Delta \boldsymbol{u}(k+j)$ is a vector of future increments of the manipulated variable, $N$ is a length of the prediction horizon, $N_{u}$ is a length of the control horizon and $\lambda$ is a weighting factor of control increments. The criterion (27) of the optimization problem can be written in a general vector form (28).

$$
J=(\hat{\boldsymbol{y}}-\boldsymbol{w})^{T}(\hat{\boldsymbol{y}}-\boldsymbol{w})+\lambda \Delta \boldsymbol{u}^{T} \Delta \boldsymbol{u}
$$

where $\boldsymbol{w}$ is a vector of the reference trajectory. The criterion can be modified using the expression to (29) and (32)-(33).

$$
J=2 \boldsymbol{g}^{T} \Delta \boldsymbol{u}+\Delta \boldsymbol{u}^{T} \boldsymbol{H} \Delta \boldsymbol{u}
$$

where the gradient $\boldsymbol{g}$ and the Hess matrix $\boldsymbol{H}$ are defined by following expressions:

$$
\begin{gathered}
\boldsymbol{g}^{T}=\boldsymbol{G}^{T}\left(\boldsymbol{y}_{0}-\boldsymbol{w}\right) \\
\boldsymbol{H}=\boldsymbol{G}^{T} \boldsymbol{G} \\
\min _{\Delta \boldsymbol{u}} 2 \boldsymbol{g}^{T} \Delta \boldsymbol{u}+\Delta \boldsymbol{u}^{T} \boldsymbol{H} \Delta \boldsymbol{u} \\
\boldsymbol{M} \Delta \boldsymbol{u} \leq \boldsymbol{\gamma} \\
\boldsymbol{M} \in \mathbb{R}^{m, n}, \boldsymbol{\gamma} \in \mathbb{R}^{m, 1} \\
\left.\left[\begin{array}{cccc}
M_{11} & M_{12} & \cdots & M_{1 n} \\
M_{21} & M_{22} & \cdots & M_{2 n} \\
\vdots & \vdots & \ddots & \vdots \\
M_{m 1} & M_{m 2} & \cdots & M_{m n}
\end{array}\right]\right\} \\
\gamma=\left[\begin{array}{llll}
\gamma_{1} & \gamma_{2} & \cdots & \gamma_{m}
\end{array}\right]^{T}
\end{gathered}
$$

Expessions (32)-(33) for determination of the desired vector of future increments of the manipulated variables has been frequently realized by Hildreth's method [7].

\section{Modified optimizer in MPC}

The modification of Hildreth's method presented in [11] was used in this paper for purposes of analysis and comparison of control quality.

This modification of the dual Hildreth's method proposes a new exit condition of the iterative numerical algorithm which is based on a comparison of two last vectors (35) in context of the dual optimization method [13] (36)-(38) using following condition (39).

$$
\left.\begin{array}{c}
\boldsymbol{d}(\omega)=\left[\begin{array}{lll}
d_{1}(\omega) & \cdots & d_{N u}(\omega)
\end{array}\right]^{T} \\
\boldsymbol{d}(\omega-1)=\left[\begin{array}{lll}
d_{1}(\omega-1) & \cdots & d_{N u}(\omega-1)
\end{array}\right]^{T}
\end{array}\right\}
$$

$$
\left.\begin{array}{c}
\boldsymbol{d}=\left[\begin{array}{lll}
d_{1} & \cdots & d_{N u}
\end{array}\right]^{T}= \\
=\arg \min \left\{\frac{1}{2} \boldsymbol{d}^{T} \boldsymbol{N} \boldsymbol{d}+\boldsymbol{o}^{T} \boldsymbol{d} \mid \boldsymbol{d} \geq \boldsymbol{0}\right\} ; \\
\boldsymbol{d} \in \mathbb{R}^{N u, 1}
\end{array}\right\}
$$




$$
\left.\begin{array}{c}
\boldsymbol{N}=\boldsymbol{M H}^{-1} \boldsymbol{M}^{T} ; N_{i j} \in \boldsymbol{N} \in \mathbb{R}^{N u, N u} ; \\
\boldsymbol{o}=\boldsymbol{M} \boldsymbol{H}^{-1} \boldsymbol{b}^{T}+\boldsymbol{\gamma} ; o_{i j} \in \boldsymbol{o} \in \mathbb{R}^{N u, 1}
\end{array}\right\}
$$

\section{Proposal of analysis of control quality using testing hypotheses}

For the purposes of evaluation of quality of control by means of analysis of discrete signals (controlled variables, increments of manipulated variables, control errors), the testing hypothesis can be utilized. The testing hypothesis can be based on a comparison of paired values of the discrete signals. A particular type of the paired values comparison corresponds to an individual type of mathematical hypothesis. It can be solved either by the Paired T-test or by the Wilcoxon Paired test. The type of the test is chosen according to the normality property of the tested data. If the data fulfil normality, the Paired T-test is used. Otherwise the Wilcoxon Paired test is then applied.

An important initial part in testing hypotheses is a declaration of the significance level $\alpha$. In technical applications, it is generally considered as strictly defined for the value of 0.001 . This value of the significance level was also applied in this paper. The second part of testing hypothesis is a declaration of the zero hypothesis. The zero hypothesis is given by the following proposition: "There are not statistically significant differences between pairs of values". An alternative hypothesis is then defined as: "There are statistically significant differences between pairs of values". The results obtained from the testing hypothesis are considered in the form of $p$ values [9]. The zero hypothesis is failed to reject, if the $p$ value of the testing hypothesis is greater or equal to $\alpha$. Zero hypothesis is rejected in favour of the alternative hypothesis if $p$ is lower than $\alpha$.

\section{Results}

By the analysis using testing hypotheses, influence of simplifications performed in the MPC algorithm was evaluated. The paired comparison statistical methods were applied. Results of the testing normality of the data by Shapiro-Wilk and Anderson-Darling tests were an assumption for selection of an appropriate statistical method. The discrete signals in multivariable MPC with constraints were analysed in the hypothesis testing with regards to quality of control.

The modified MPC was compared with the nonmodified MPC by simulation with constrained optimization problem in MATLAB. Following TITO model (40)-(41) was being controlled. The setting of MPC's parameters (42) and conditions (43) were considered. The matrix $\boldsymbol{I}$ is an identity matrix and $\boldsymbol{E}$ is a unit matrix.

$A\left(z^{-1}\right)=\left[\begin{array}{cc}1-1.3264 z^{-1}+0.3271 z^{-2} & 0.024 z^{-1}-0.0029 z^{-2} \\ -0.0711 z^{-1}+0.0759 z^{-2} & 1-1.0911 z^{-1}+0.134 z^{-2}\end{array}\right]$

$\boldsymbol{B}\left(z^{-1}\right)=\left[\begin{array}{cc}0.2983 z^{-1}-0.097 z^{-2} & 0.093 z^{-1}+0.0682 z^{-2} \\ 0.1755 z^{-1}+0.0688 z^{-2} & 0.1779 z^{-1}+0.1065 z^{-2}\end{array}\right]$

The obtained simulation results are presented in Figure 1-4.

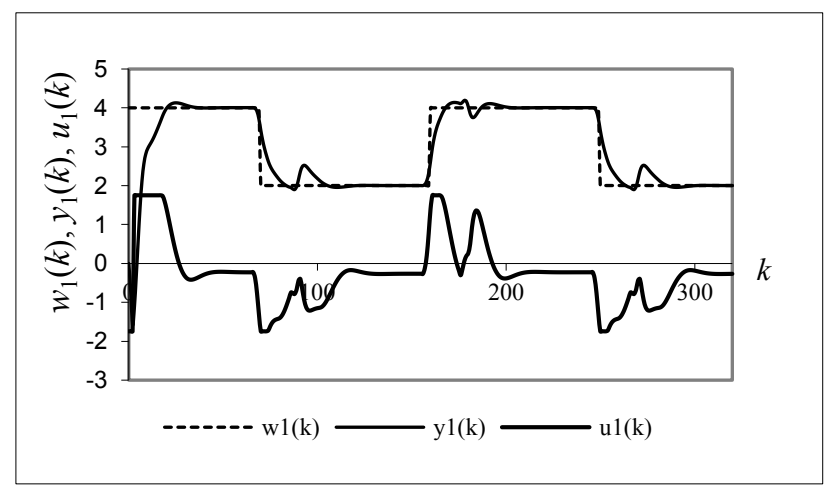

Fig. 1. Simulation of MPC without Modifications $-1^{\text {st }}$ Variables of Multivariable Control. 


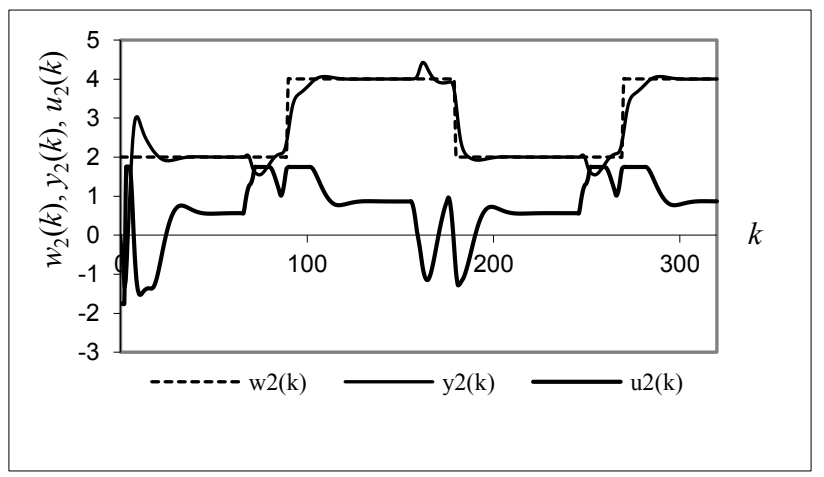

Fig. 2. Simulation of MPC without Modifications $2^{\text {nd }}$ Variables of Multivariable Control.

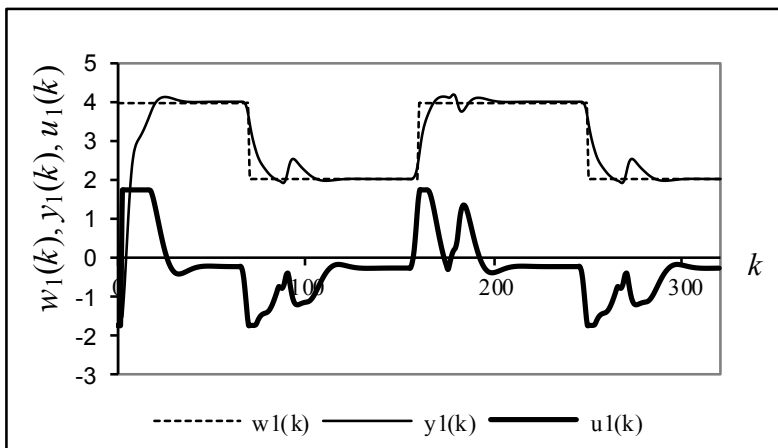

Fig. 3. Simulation of MPC with Modification - $1^{\text {st }}$ Variables of Multivariable Control.

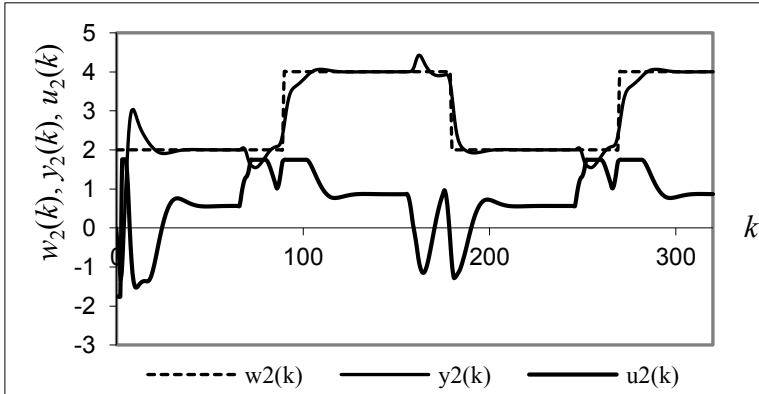

Fig. 4. Simulation of MPC with Modification $2^{\text {nd }}$ Variables of Multivariable Control.

As can be seen in Table 1, values of control quality criterions (1)-(2) were slightly decreased and the lower number of floating point operations was measured in MATLAB using rules [12].

Table 1. Decreasing of number of operations in analyzed modified MPC with influences on control quality.

\begin{tabular}{|c|c|c|c|c|c|c|}
\cline { 2 - 7 } \multicolumn{1}{c|}{} & \multicolumn{3}{c|}{ Non-Modified MPC } & \multicolumn{3}{c|}{ Modified MPC } \\
\hline$N_{2}=N_{u}$ & $J_{1}$ & $J_{2}$ & $\begin{array}{c}F \\
\text { [flops] }\end{array}$ & $J_{1}$ & $J_{2}$ & $F^{*}$ [flops] \\
\hline \hline 5 & 92.333 & 268.446 & $2 . \mathrm{E}+07$ & 92.333 & 268.446 & $1 . \mathrm{E}+07$ \\
10 & 88.476 & 262.159 & $2 . \mathrm{E}+08$ & 88.477 & 262.159 & $1 . \mathrm{E}+08$ \\
15 & 87.664 & 261.577 & $8 . \mathrm{E}+08$ & 87.664 & 261.577 & $4 . \mathrm{E}+08$ \\
20 & 87.585 & 261.563 & $2 . \mathrm{E}+09$ & 87.585 & 261.563 & $1 . \mathrm{E}+09$ \\
25 & 87.588 & 261.542 & $5 . \mathrm{E}+09$ & 87.588 & 261.542 & $2 . \mathrm{E}+09$ \\
30 & 87.607 & 261.458 & $1 . \mathrm{E}+10$ & 87.607 & 261.458 & $4 . \mathrm{E}+09$ \\
35 & 87.649 & 261.335 & $2 . \mathrm{E}+10$ & 87.649 & 261.335 & $7 . \mathrm{E}+09$ \\
\hline
\end{tabular}

Using the proposed approach based on the analysis of signals between two realized versions of MPC, results (Table 2) in the form of $p$-values were achieved using the corresponding statistical tests according to the datanormality condition.

The zero hypothesis was given by the following proposition: "There are not statistically significant differences between pairs of signal values". An alternative hypothesis was then defined as: "There are statistically significant differences between pairs of signal values". Significance level was considered as 0.001 .

Table 2. Statistical analysis of pairs of signals.

\begin{tabular}{|c|c|c|c|c|}
\hline $\begin{array}{c}\text { Testing } \\
\text { Hypothesis }\end{array}$ & $\begin{array}{c}\text { Signals } \Delta u \\
\text { of both } \\
\text { Versions } \\
\text { of } M P C\end{array}$ & $\begin{array}{c}\text { Signals } u \\
\text { of both } \\
\text { Versions } \\
\text { of MPC }\end{array}$ & $\begin{array}{c}\text { Signals } \Delta e \\
\text { of both } \\
\text { Versions } \\
\text { of MPC }\end{array}$ & $\begin{array}{c}\text { Signals } y \\
\text { of both } \\
\text { Versions } \\
\text { of MPC }\end{array}$ \\
\hline \hline Results & $\begin{array}{c}p>0.001 \\
\text { Wilcoxon } \\
\text { Test }\end{array}$ & $\begin{array}{c}p>0.001 \\
\text { Wilcoxon } \\
\text { Test }\end{array}$ & $\begin{array}{c}p>0.001 \\
\text { Wilcoxon } \\
\text { Test }\end{array}$ & $\begin{array}{c}p>0.001 \\
\text { Wilcoxon } \\
\text { Test }\end{array}$ \\
\hline
\end{tabular}

According to the achieved results in Table 2, each zero hypothesis was failed to reject on the significance level 0.001. Therefore statistical significant differences between pairs of signals were not identified on the strictly declared significance level. This corresponds with the results of control quality criterions in Table 1, where only slight diferences can be observed.

\section{Conclusions}

For the purpose of application of testing hypothesis into a field of analysis of signals, the control quality achieved by two different algorithms was analyzed and compared using testing hypothesis. In this hypothesis testing, partial values of signals were analyzed in each sampling period of MPC. It would not be possible to consider statistical significance of differences in achieved control quality only from the descriptive attributes given by standardly used control quality criterions. By using of methods of testing hypotheses on existence of the statistical significant differences between two discrete signals, analysis of a control quality was successfully complemented by more mathematically supported results. The paired comparison was performed by the Wilcoxon paired test on the strictly defined significance level 0.001 . Therefore, the achieved results had relevant informational value based on mathematical statistics. It was proved that the difference between control quality of modified and original methods of optimization in predictive control was not statistically significant.

\section{References}

1. J.P. Corriou, Process control: theory and applications (Springer, 2004)

2. W.H. Kwon, Receding horizon control: model predictive control for state models (Springer, 2005)

3. E.F. Camacho, C. Bordons, Model predictive control (Springer, 2007) 
4. J.A. Rossiter, Model Based Predictive Control: a Practical Approach (CRC Press, 2003)

5. M. Kubalcik, V. Bobal, T. Barot, WSEAS Transactions on Systems and Control 13 (2018)

6. D. Ingole, J. Holaza, B. Takacs, M. Kvasnica, 20th International Conference on Process Control (PC). IEEE (2015)

7. L. Wang, Model Predictive Control System Design and Implementation Using MATLAB (Springer, 2009)

8. M. Kubalcik, V. Bobal, JMechE Part I : Systems and Control Engineering 220 (2006)

9. B. Kitchenham, et.al., Empirical Software Engineering (2016)

10. M. Vaclavik, Z. Sikorova, T. Barot, Computational and Statistical Methods in Intelligent Systems, Advances in Intelligent Systems and Computing, 859, Springer (2019)

11. M. Kubalcik, V. Bobal, T. Barot, Innovation, Engineering and Entrepreneurship, Lecture Notes in Electrical Engineering 505 (2019)

12. R. Hunger, Technical Report [online]. Technische Universität München, Associate Institute for Signal Processing (2007)

13. Z. Dostal, Optimal Quadratic Programming Algorithms: With Applications to Variational Inequalities (Springer, 2009) 